\title{
Effect of Human Crowd Obstruction on the Performance of an Urban Small-Cell Millimeter- Wave Access Network
}

\author{
Mohammed Zahid Aslam, Yoann Corre \\ SIRADEL \\ Rennes, France
}

\author{
Yves Lostanlen \\ SIRADEL North America \\ Toronto, Ontario, Canada \\ yves.lostanlen@ieee.org
}

\begin{abstract}
Hybridation between ray-based deterministic propagation modelling and statistical components is viewed as a promising solution to assess the performance of outdoor smallcell network designs or allocation techniques. In the solution presented in this paper, the main properties of the propagation channel (i.e. the specular paths, and the blockage by large objects) are simulated from the 3D geographical data and the ray-based model, while the effect of small or dynamic in-street objects, like vehicles and human bodies, can be appropriately reproduced by a stochastic procedure. A new feature of the solution consists in the creation of random clusters of human bodies in the surrounding of the users (the impact of the user body itself was previously introduced in the hybrid model as a stochastic factor). The application and interest of the new feature is illustrated in a radio access $60-\mathrm{GHz}$ small-cell scenario, where small-cells are installed on lampposts and users are distributed in the street. The blockage by the human bodies affects the cell selection and beam orientation; the degradation of the service coverage is evaluated based on the signal-to-noise ratio (SINR) and for various human crowd densities.
\end{abstract}

Keywords-millimeter-wave, ray-tracing, human body obstructions, small-cell network.

\section{INTRODUCTION}

Stochastic propagation channel models have been so far a popular solution for the multilink design, evaluation and validation of the radio-cellular systems including $3 \mathrm{G}$ and $4 \mathrm{G}$. These models have evolved from the simple tapped delay line models to the MIMO-compliant geometry-based bidirectional models like the ones developed in the European COST 273 action [1] or the WINNER projects [2][3]. On the other hand, the ray-tracing or ray-launching based deterministic approaches were used for highly complex real environments to perform radio-planning and network coverage simulations; much later in the process from the design to the deployment of the systems. This approach was well suited for sub- $6 \mathrm{GHz}$ systems where the electromagnetic wave penetration was not a major concern. Therefore, in the initial phases of the design and evaluation of networks, rapid simulations could be made utilizing the stochastic approaches.

In millimetre-wave (mmWave) small-cell networks, the channel properties strongly differ from those at sub-6GHz and highly detailed environmental factors have to be considered. In particular, mmWaves are not able to penetrate through or get around the obstacles in the same way as at sub-6 $\mathrm{GHz}$. Therefore, detailed descriptions of the environment including 3D obstacles like buildings but also vegetation (from large blocks of vegetation to isolated trees), large vehicles, urban furniture and even human bodies have to be considered. Deterministic ray-based approaches provide the support for channel models considering physically fixed obstacles like buildings and detailed vegetation that can be obtained through map data. However, the deterministic approach is not an ideal solution in case of obstacles that are not static or fixed permanently to a location like vehicles and human bodies.

Hybrid ray-based models provide the solutions utilizing both the advantages from the stochastic and deterministic channel models. In these models, it is possible to utilize the map data with high details of the environment, even individual trees, through a deterministic ray-based model. Other nonstatic in-street obstacles like large vehicles and human bodies can be modelled using stochastic approaches in the same model. The recent METIS channel model [4] proposed a mapbased approach in which complete geographic data (so-called Madrid grid) is used along with ray-based predictions to obtain the channel properties. In a hybrid approach, such a model can be used to generate some large-scale parameters from the deterministic model and then feed this data into a stochastic model to get a better perspective of the channel. Stochastic approaches to model blockage due to the human body have been used in [5] [6]. Other recent models, such as the one proposed by the European project MiWEBA also utilize a hybrid approach for some deterministic reflected paths along with other stochastic components [7].

The authors have already presented several evolutions in the propagation model integrated in their mmWave network simulator: vegetation effect [8], vehicle obstruction and user self-obstruction [9]. Conclusions from the previous studies [8] [9] show that there is only a small impact of in-street obstacles like large vehicles and user-body self-obstruction. This small impact is achieved by simple mechanisms like an optimal cell selection process and physically placing the base stations asymmetrically. Apart from these, the blockage itself causes a 
positive impact by reducing the interference level by better inter-cell isolation.

This work is continued along with the introduction of human crowd obstruction, in a stochastic way, combined with the deterministic ray-based channel prediction and existing user self-obstruction model. The modelling approach is detailed in section II.

By using this enhanced propagation model, Section III of the paper aims at evaluating the effect of various human traffic densities on a $60-\mathrm{GHz}$ small-cell network performance: degradation of the service coverage and SINR.

Section IV draws some conclusions and gives perspective on this still on-going work.

\section{HUMAN CROWD OBSTRUCTION MODELLING}

The objective of the work done in this paper is to evaluate the effect of human obstruction due to a crowd of people surrounding a user. In [9], the impact of the human body selfobstruction was studied for mmWave networks. Selfobstruction is caused due to the user's body that could obstruct the multipath propagation degrading not just the received power but also the interference, which is desirable. Since the human body self-obstruction is omnipresent, it is used in all simulations conducted in this work as well.

The diffraction caused by the human body has been widely studied in the literature using the Knife Edge Diffraction (KED) models [4][8][9][10]. The recent METIS model [4] proposed the use of the Double Knife Edge Diffraction (DKED) model by considering the human body as an infinitely long screen with two edges.

In the case of the user body self-obstruction, since the antenna is located very close to the body, it is seen that the KED model underestimates the shadowing. The Uniform Theory of Diffraction (UTD) based models like the creeping wave model have shown to perform well as compared to the exact solution [14], and is proposed for on-body channel predictions.

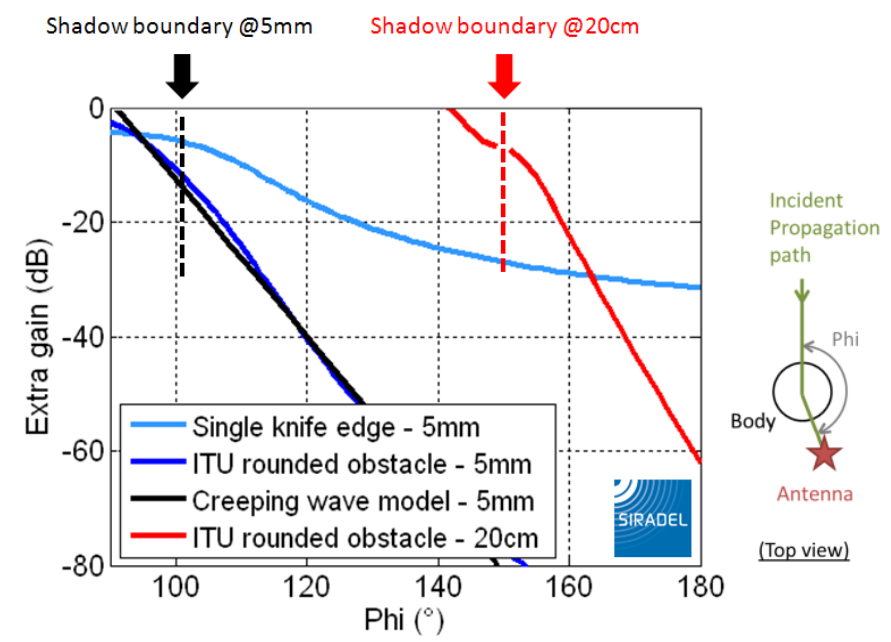

Fig. 1. Comparison between different models for prediction of the user self-obstruction.
For implementation simplicity, the rounded edge model by ITU-526 [13] has been used to model the diffraction caused by the user body self-obstruction. This model takes into consideration the additional loss incurred due to a rounded obstacle, which is added to the KED loss. The relevance of this model is well-known for large range propagation and was evaluated for short range obstructions in [9]. Fig. 1 shows the relation between the KED models as compared with the ITU rounded obstacle and Creeping Wave model with $5 \mathrm{~mm}$ separation between the user body and the antenna. Clearly, for cases in which the antenna is close to the user body, the ITU rounded edge model gives better results which are comparable to the accurate Creeping Wave model.

The user body is considered to be a perfectly conducting uniform cylinder [9]. The radius of the cylinder used is $0.2 \mathrm{~m}$. A shadow region is calculated based on the arrival angle of the incident path and the distance of the user equipment from the surface of the body. To model the human bodies as a crowd surrounding the user, groups of human bodies (clusters) of different sizes are created. Each cluster of a given size is assumed to contain a certain number of closely located human bodies and the cluster itself is considered to be an opaque obstruction. Diffraction occurs at the side and the top of the cluster. The KED model is used in case of the clusters as the receiver is located further away from the cluster as compared to the user body self-obstruction case. The average height of the crowd clusters is considered to be $1.7 \mathrm{~m}$ and the user equipment is assumed to be located at $2 / 3$ of the average height for e.g. user holding a smartphone. Reflection and scattering on the bodies is not considered.

The human crowd obstruction is applied on the direct path and all multi-paths initially generated by the ray-based simulation, as illustrated in Fig. 2. The clusters surrounding the user are generated in a random way and (in current version of the implementation) independently from one user to the other. The shadow region created by each cluster depends on the distance between the user antenna and the cluster, size of the cluster and the difference between the heights of the antenna and cluster.

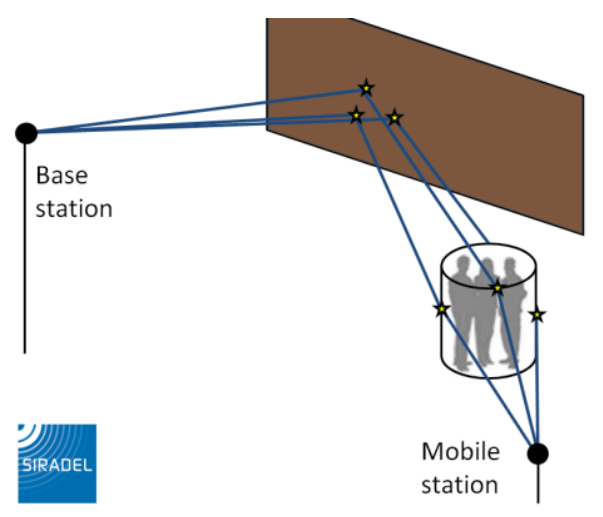

Fig. 2. Example of obstruction of an initial reflected path by a body cluster, leading to three diffacted paths (from top and sides of the cluster).

Fig. 3 shows the relation between the horizontal shadow region and the distance to the antenna for various cluster sizes (radii). As the distance between the cluster and the antenna 
increases the shadow region significantly becomes smaller. Therefore, for a cluster size of radius $1.0 \mathrm{~m}$ the shadow region is less than $40^{\circ}$, which is just $11.1 \%$ of the horizontal plane. Further, when the impact of the vertical direction is also added this distance reduces further. In our simulations, the impact due to the clusters beyond $4 \mathrm{~m}$ is very small especially in case of the smaller sized clusters.

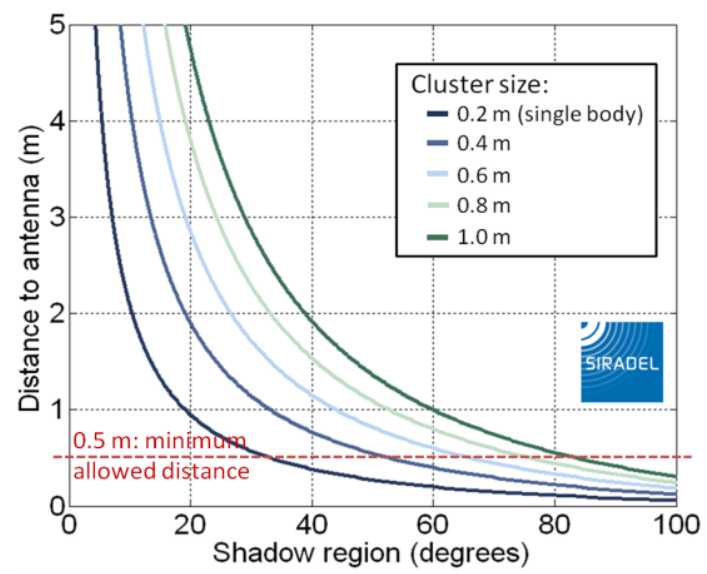

Fig. 3. Shadow region generated by the body cluster, depending on its size and distance to the user antenna.

A random number of clusters are stochastically positioned around the user using a Poisson process based random variable generator. Fig. 4 illustrates how the clusters impact the Power Delay Profile of the two signals S1 and S2 captured by the same user equipment but coming from two different base stations. The multi-path propagation channels are composed of several contributions arriving with specific delays and angles. The power of the paths arriving in the shadow region (or more precisely: in the close vicinity of the shadow region, as significant diffraction loss occurs even without optical path obstruction) are reduced in a similar way for both signals.

The Poisson parameter $(\lambda)$ which is the expected number of occurrences is varied to achieve different crowd densities. The following scenarios are considered.

Very crowded scenario: the crowd is relatively dense in close vicinity of the user. It is representative of open-air events, concerts, crowds created during arrival and departure of flights, trains etc. Larger sized clusters located around $2 \mathrm{~m}$ from the user are considered.

Moderately crowded scenario: the crowd is moderately dense and located at a larger distance from the user. This scenario is representative of crowded shopping streets, public beaches, main road pedestrian intersections etc. The simulations are conducted such that medium to large sized clusters are considered in a range of around $3 \mathrm{~m}$ from the user.

Crowded scenarios: the crowd is sparse and can be located at a distance away from the receiver. This could be in the case of users waiting at a bus stop, tourist spots, waiting areas of airports or train stations, universities, hospitals etc. The simulations are conducted with small to medium sized clusters located up to $4 \mathrm{~m}$ away from the receiver.

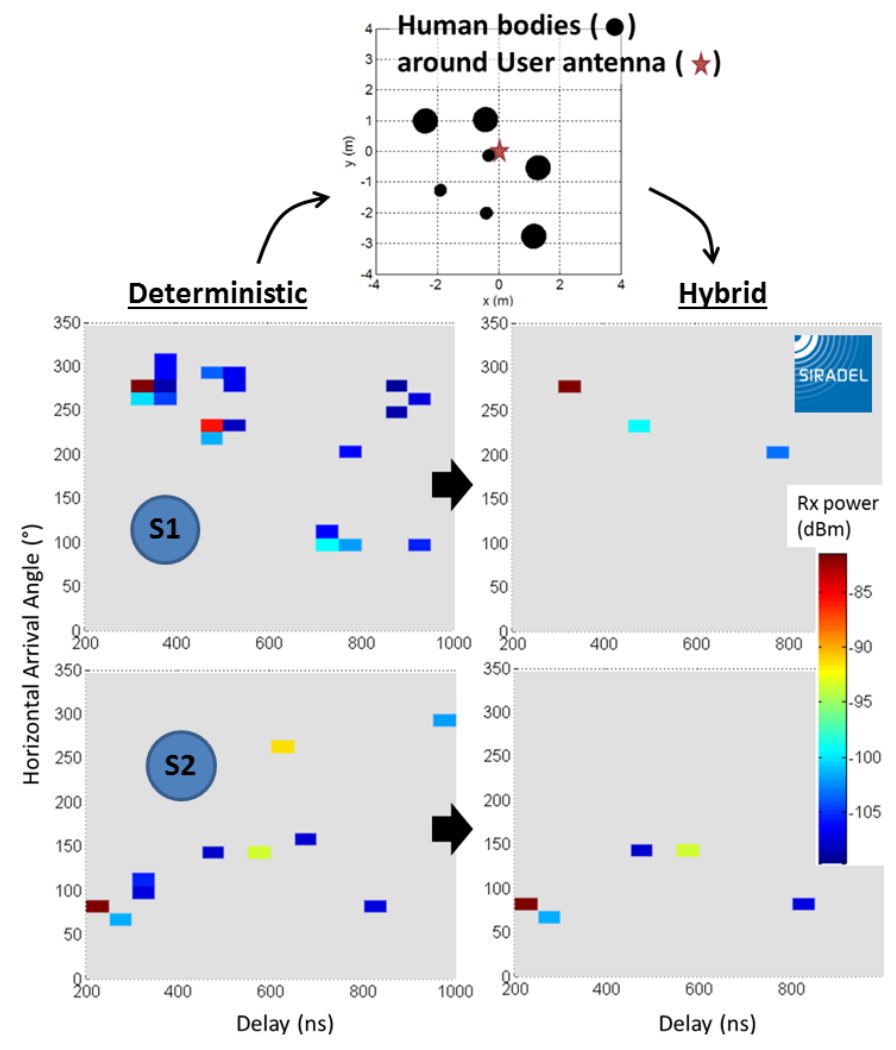

Fig. 4. Examples of two signals S1 and S2 from different base stations to the same user antenna. They are obstructed by the same body clusters. Initial Angular Power Delay Profile of the channel is given by the ray-based model, but is modified by the application of the stochastic human crowd obstruction.

\section{NETWORK SIMULATION RESULTS}

\section{A. Simulation principles and Scenario}

To simulate the impact of the human body obstruction caused by people in the near vicinity of the user, a small outdoor area containing two small-cells is considered, shown in Fig. 5. The selected area is part of a larger small-cell network that was designed in [8] to obtain seamless outdoor coverage. The distance between the two small-cells is $80 \mathrm{~m}$. They are located on in-street lampposts at a height of $7 \mathrm{~m}$ above the ground. The half-power beamwidth (HPBW) of the automatically steerable transmit antenna at the small-cell is $22^{\circ}$ with a maximum gain of $18.5 \mathrm{dBi}$. The transmit power is adjusted for an EIRP (Equivalent Isotropic Radiated Power) of $40 \mathrm{dBm}$.

Users of height $1.7 \mathrm{~m}$ with a finite throughput demand are dropped along the sidewalks shown by the blue lines in Fig. 5. A Monte Carlo process is used to drop random users, run network simulations and extract the performance statistics with or without the impact of the human-body obstruction. Multipath propagation is considered along with suitable cell and beam selection. The interference levels are calculated based on all the users served by the neighbour cell, at each iteration as described in detail in [9]. 


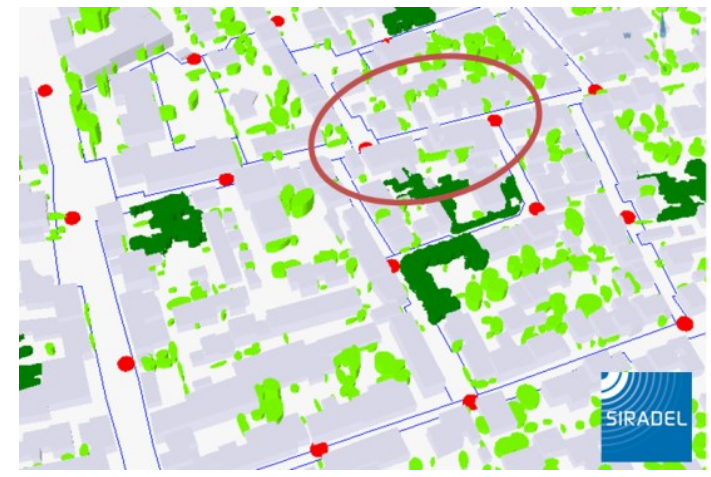

Fig. 5. Small-cell deployment (red dots) and user locations (sidewalks: blue lines) in real European city environment. The small-cells considered in this study are encircled.

The user body is randomly oriented at each iteration, to include the self-obstructing impact. The clusters are generated around each user driven by parameters given in Table I. The positions for the different crowd densities in one Monte Carlo iteration are shown Fig. 6.

TABLE I. SIMULATION PARAMETERS

\begin{tabular}{|c|c|}
\hline System & $\begin{array}{l}\text { - PHY waveform: Single-Carrier (SC) } \\
\text { - Central frequency: } 60 \mathrm{GHz} \\
\text { - Bandwidth: } 200 \mathrm{MHz} \\
\text { - Mapping table: from IEEE } 802.11 \mathrm{ad} \text {, with } 12 \\
\text { adaptative modulation schemes (MCS) } \\
\text { - PHY net data rate: } 43.8 \text { to } 525.0 \mathrm{Mbps}\end{array}$ \\
\hline Users & $\begin{array}{l}\text { - Noise figure: } 7 \mathrm{~dB} \\
\text { - Antenna: omni-directional, } 5 \mathrm{dBi} \\
\text { - Demand : } 15 \mathrm{Mbps} \\
\text { - Active user density: } 1000 \text { users } / \mathrm{km}^{2}\end{array}$ \\
\hline $\begin{array}{l}\text { Power } \\
\text { budget }\end{array}$ & $\begin{array}{l}\text { - Impairment loss: } 8 \mathrm{~dB} \\
\text { - DL Rx sensitivity: }-79 \text { to }-64 \mathrm{dBm}\end{array}$ \\
\hline $\begin{array}{l}\text { Human } \\
\text { crowd }\end{array}$ & $\begin{array}{l}-\lambda(\text { radius }=0.2 \mathrm{~m})=6 \\
-\lambda(\text { radius }=0.4 \mathrm{~m})=4 \\
-\lambda(\text { radius }=0.8 \mathrm{~m})=2 \\
- \text { Max range from user antenna }=2 \mathrm{~m} \text { (very } \\
\text { crowded), } 3 \mathrm{~m} \text { (moderately crowded), } 4 \mathrm{~m} \\
\text { (crowded) }\end{array}$ \\
\hline Other & $\begin{array}{l}\text { - Monte-Carlo: } 30 \text { iterations } \\
\text { - Traffic: Non full-buffer }\end{array}$ \\
\hline
\end{tabular}

\section{B. Impact of human crowd obstruction}

The effect of the user-body obstruction is evaluated through system level simulations considering both the user body self-obstruction and the impact due to the different sizes of crowds. The network performance is assessed in five different situations: 1) no body obstruction (not realistic); 2) self-body obstruction; 3-5) self-body and crowd obstruction with different crowd densities. The area including the two small-cells marked in Fig. 5 is considered. In the first step, the deterministic predictions of the channel are obtained using a ray-based approach with multipath propagation. Due to the street-canyon like nature of the scenario a rich multipath environment is created. These predictions are then fed into a stochastic system level simulator which uses a Monte Carlo scheme. Users are randomly dropped along the sidewalks (marked in blue lines in Fig. 5) in the selected area. In Fig. 6, the positions for the different crowd densities in a single iteration are shown. In the case of the very crowded scenario, large sized clusters in a dense environment are located very close to the mobile station. In case of the moderately crowded scenario, the clusters are located further away and are composed mostly of medium sized clusters. Finally, in case of the crowded scenario, small to medium sized clusters are mostly considered located at a distance which is even further from the mobile station. The user body is randomly oriented at each iteration, to include the self-obstructing impact.

The channel performance is evaluated by considering the three different crowd densities along with the impact in the absence of any crowd but only with the user body selfobstruction and finally without consideration of any human body blockage (purely deterministic). The Cumulative Distributive Function (cdf) results for the SINR and the Intercell Interference (ICI) levels is shown in Fig. 7 and 8.
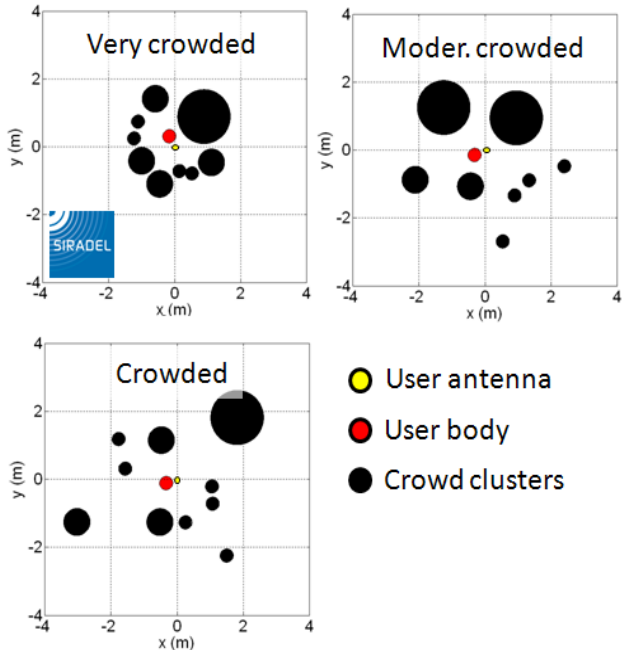

Crowd clusters

Fig. 6. Different crowd density scenarios simulated

The inter-cell interference (ICI) reduces as the number and size of the obstacles is increased in the scenario. This can be seen in the Fig. 7, in which the probability of interference levels is the least for the case without the human-body obstructions. At the median value, the interference level without the human body obstruction is $-86.4 \mathrm{dBm}$ whereas for the very crowded scenario it is $-93.5 \mathrm{dBm}$. This is due to the fact that the clusters, especially large ones in the case of a very crowded scenario are located very close to the mobile station which causes large shadow region that not only block the signal but also the interference. This can be seen as a positive impact due to the blockages. At lower percentiles, the very crowded scenario and the moderately crowded scenario have similar interference levels whereas the other obstruction scenarios tend to the no human body obstruction levels.

For the three different crowd scenarios, it can be seen from Fig. 8 that the SINR values reduce as the density of the crowd increases. The impact of only the user-body self-obstruction as compared to the one without any human blockage is quite limited which is a result of the lower interference levels 
(positive impact) and the process of optimal cell selection, used in the simulations. The $10^{\text {th }}$ percentile value of the SINR without human body obstruction is $7.9 \mathrm{~dB}$ whereas it is -2.6 $\mathrm{dB}$ at the densely crowded scenario. Therefore, even with the optimal cell selection process and the reduction of the interference there still exists a reduction in SINR by $10.5 \mathrm{~dB}$. For a service that requires SINR $>0 \mathrm{~dB}$, the difference in percentiles between the extremes of not considering any human bodies (deterministic) vs. the densely crowded obstruction is $7.8 \%$ (from $4.7 \%$ to $12.5 \%$ ). This means that just due to the addition of the human body obstruction (self and dense crowd), an extra $7.8 \%$ users may be denied service. This can be seen as a significant impact of blockage due to the human body obstruction, which must be considered in the channel modelling for mmWave dense small-cell networks.

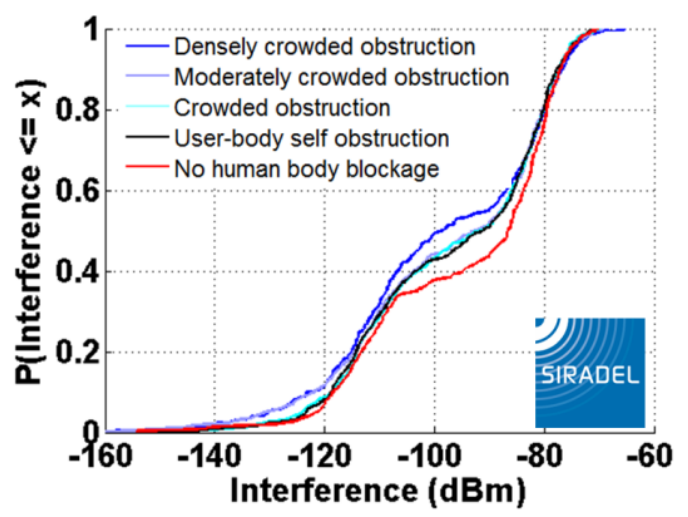

Fig. 7. Human body obstruction: CDF of Inter-cell Interference levels.

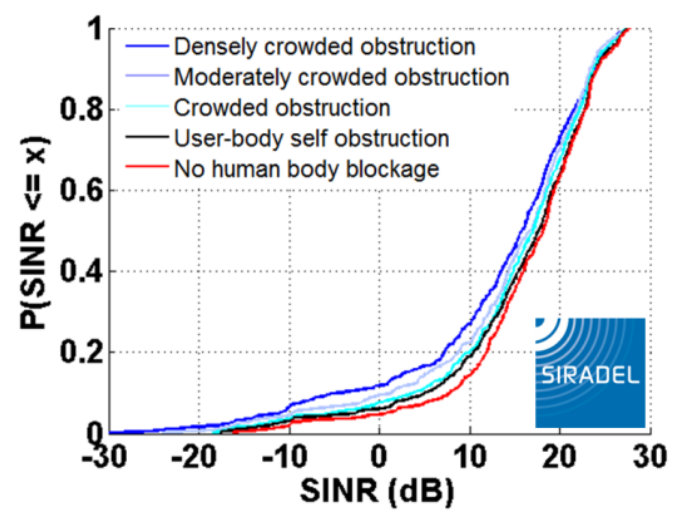

Fig. 8. Human body obstruction: CDF of SINR.

\section{CONCLUSIONS AND PERSPECTIVES}

A hybrid ray-based model is applied for the evaluation of mmWave channels using the deterministic approach for largescale fixed obstructions and a stochastic approach for random non-static objects. The goal of this on-going study is to produce realistic channel realizations in the context of the evaluation of dense mmWave small-cell networks. The presented study evaluates the obstruction by the human body. The obstruction is of two types; one caused by the user-body itself and the other is the obstruction due to the crowd of people surrounding the user. A random crowd distribution following a Poisson process is generated around a user along with random orientations of the user equipment itself (for selfblockage).

The human body obstruction as compared to no human body obstruction causes significant degradation of $2.8 \mathrm{~dB}$ and $1.5 \mathrm{~dB}$ in case of densely and moderately crowded scenarios respectively. This impact can be reduced by physically placing the antennas at higher and asymmetric positions. Also, the optimal cell selection process helps to reduce the blockage impact. Hybrid ray-based models with stochastic components are at an early stage and measurements are required for further improvement and calibration of these models. The proposed model should later be extended to scenarios with mobility in which the users are moving in between the small-cells. Adding dynamic dimensions (i.e. time variant channel and network scheduling) remains a major perspective.

\section{ACKNOWLEDGMENT}

This work is supported by the European Commission through the H2020-ETN-5Gwireless project under grant 641985.

\section{REFERENCES}

[1] COST 273, Mobile Broadband Multimedia Networks - Techniques, Models and Tools for 4G, Edited by L. Correia, Published by Elsevier Ltd, 2006.

[2] IST-WINNER II project, WINNER II Channel Models, Deliverable D1.1.2, v1.2, 2007.

[3] CELTIC-WINNER+ project, WINNER+ Final Channel Models, Deliverable D5.3, v1.0, 2010.

[4] ICT-METIS project, METIS Channel Models, Deliverable D1.4, 2015.

[5] T. Bai and R. W. Heath, "Analysis of self-body blocking effects in millimeter wave cellular networks," in 48th Asilomar Conference on Signals, Systems and Computers, Pacific Grove, CA, 2014.

[6] M. Abouelseoud and G. Charlton,"The Effect of Human Blockage on the Performance of Millimeter-Wave Access Link for Outdoor Coverage," in IEEE 77th Vehicular Tech Conf (VTC), Dresden, 2013.

[7] ICT-MiWEBA project, Channel Modeling and Characterization, Deliverable D5.1, v1.0, 2014.

[8] Y. Corre, R. Charbonnier, M. Z. Aslam and Y. Lostanlen, "Assessing the performance of a 60-GHz Dense Small-Cell Network Deployment from Ray-Based Simulations", $21^{\text {st }}$ IEEE CAMAD workshop, Toronto, Canada, 2016.

[9] R. Charbonnier, M. Z. Aslam, Y. Corre and Y. Lostanlen, "Mixing Deterministic and Stochastic Propagation for Assessing MmWave Small-cell Networks", in 11th IEEE European Conference on Antennas and Propagation (EuCAP), Paris, France, 2017.

[10] M. Jacob, S. Priebe, T. Kurner, M. Peter, M. Wisotzki, R. Felbecker, and W. Keusgen, "Fundamental analyses of $60 \mathrm{GHz}$ human blockage," in 7th IEEE European Conference on Antennas and Propagation (EuCAP), Göteborg, Sweeden, 2013.

[11] Y. Corre, M. Brau and Y. Lostanlen, " $3 \mathrm{G}$ Femtocell wireless timevariant stochastic channel modelling related to indoor human activity", $22^{\text {nd }}$ IEEE PIMRC symposium, Toronto, Canada, 2011.

[12] IEEE Std 802.11ad, Wireless LAN Medium Access Control (MAC) and Physical Layer (PHY) Specifications, Amendment 3: Enhancements for Very High Throughput in the $60 \mathrm{GHz}$ Band, 2012.

[13] ITU-R P.526-13, Propagation by diffraction, 2013

[14] T. Mavridis, L. Petrillo, J. Sarrazin, D. Lautru, A. Benlarbi-Delai, and P. De Doncker, "Theoretical and experimental investigation of a 60- ghz off-body propagation model," IEEE Trans on Ant and Prop, Jan 2014. 